\title{
High-resolution continuum polarization measurements in the near-infrared to submillimeter wavelength range
}

Sebastian Wolf, Bringfried Stecklum, Thomas Henning, Ralf Launhardt, Hans Zinnecker

Sebastian Wolf, Bringfried Stecklum, Thomas Henning, Ralf Launhardt, Hans Zinnecker, "High-resolution continuum polarization measurements in the near-infrared to submillimeter wavelength range," Proc. SPIE 4843, Polarimetry in Astronomy, (14 February 2003); doi: 10.1117/12.458863

SPIE Event: Astronomical Telescopes and Instrumentation, 2002, Waikoloa, Hawai'i, United States 


\title{
High-resolution Continuum Polarization Measurements in the Near-infrared to Submillimeter Wavelength Range
}

\author{
S. Wolf ${ }^{a}$, B. Stecklum ${ }^{b}$, Th. Henning ${ }^{c}$, R. Launhardt ${ }^{d}$, and H. Zinnecker ${ }^{e}$ \\ ${ }^{a}$ California Institute of Technology, 1200 E California Blvd, Pasadena, CA 91125, USA \\ ${ }^{b}$ Thüringer Landessternwarte Tautenburg, Sternwarte 5, 07778 Tautenburg, Germany \\ ${ }^{c}$ Max-Planck-Institut für Astronomie, Königsstuhl 17, 69117 Heidelberg, Germany \\ ${ }^{d}$ Division of Physics, Mathematics and Astronomy, \\ California Institute of Technology, MS 105-24, Pasadena, CA 91125, U.S.A. \\ ${ }^{e}$ Astrophysikalisches Institut Potsdam, An der Sternwarte 16, D-14482 Potsdam, Germany
}

\begin{abstract}
We present high-resolution polarization maps, obtained with near-infrared instruments such as ISAAC at the VLT, SOFI at the NTT, and SCUBA at the JCMT. While we use the near-infrared polarization maps to determine the structure of the optical reflection nebula Cederblad 110 IRS 4 and to investigate the alignment of circumstellar disks around T Tauri binary stars, submillimeter polarization maps are used to derive the magnetic field structure and strength in Bok globules. Furthermore we show that near-infrared polarimetry represents a powerful tool to distinguish between different polarization models developed for active galactic nuclei.
\end{abstract}

Keywords: Polarization, Scattering, Dichroic extinction, Grain alignment, Circumstellar dust, Magnetic field, Bok globules, Young stellar object, Binaries, Active Galactic Nucleus (AGN), Radiative transfer

\section{INTRODUCTION}

Polarized light is produced by dust grains, such as in the interstellar medium or the vicinity of young stellar objects, through the following mechanisms:

1. Light scattering

This mechanism is most important in the near-infrared and shorter wavelength range. If scattering by (nearly) spherical or mis-aligned elongated dust grains dominates, the polarization vectors are oriented perpendicular to the radius vector towards the illuminating source. As illustrated in Fig. 1, this allows the determination of the location of the illuminating sources in reflection nebulae. ${ }^{1,2}$ However, deviations from the centro-symmetric polarization pattern can be caused by multiple illuminating sources and/or scattering by aligned non-spherical grains. ${ }^{3}$ Examples, in particular a polarization survey of low-mass young pre-main sequence stars and a theoretical study on the polarization of active galactic nuclei (AGNs), are discussed in Sect. 3 and 4.

2. Thermal radiation and dichroic absorption by aligned dust grains

In the dusty envelopes around young stellar objects, polarization due to dichroic extinction and thermal emission by spinning dust grains is the most important signature of magnetic fields. ${ }^{6-8}$ Furthermore, the large-scale magnetic field structure in the interstellar medium can be traced by the interstellar polarization resulting from dichroic extinction of background starlight by aligned interstellar dust grains. ${ }^{9}$ Non-spherical dust grains are expected to be aligned by the following mechanisms: Paramagnetic relaxation, ${ }^{10-12}$ supersonic flows ${ }^{13,14}$ and/or radiative torques. ${ }^{15,16}$ Irrespective of the alignment mechanism, charged interstellar grains would have a substantial magnetic moment, leading to a rapid precession of the grain angular momentum $\vec{J}$ around the magnetic field direction $\vec{B}$ (see Draine \& Weingartner ${ }^{16}$ and

Further author information: (Send correspondence to S. Wolf)

S. Wolf: E-mail: swolf@ipac.caltech.edu, Telephone: 16263958794 


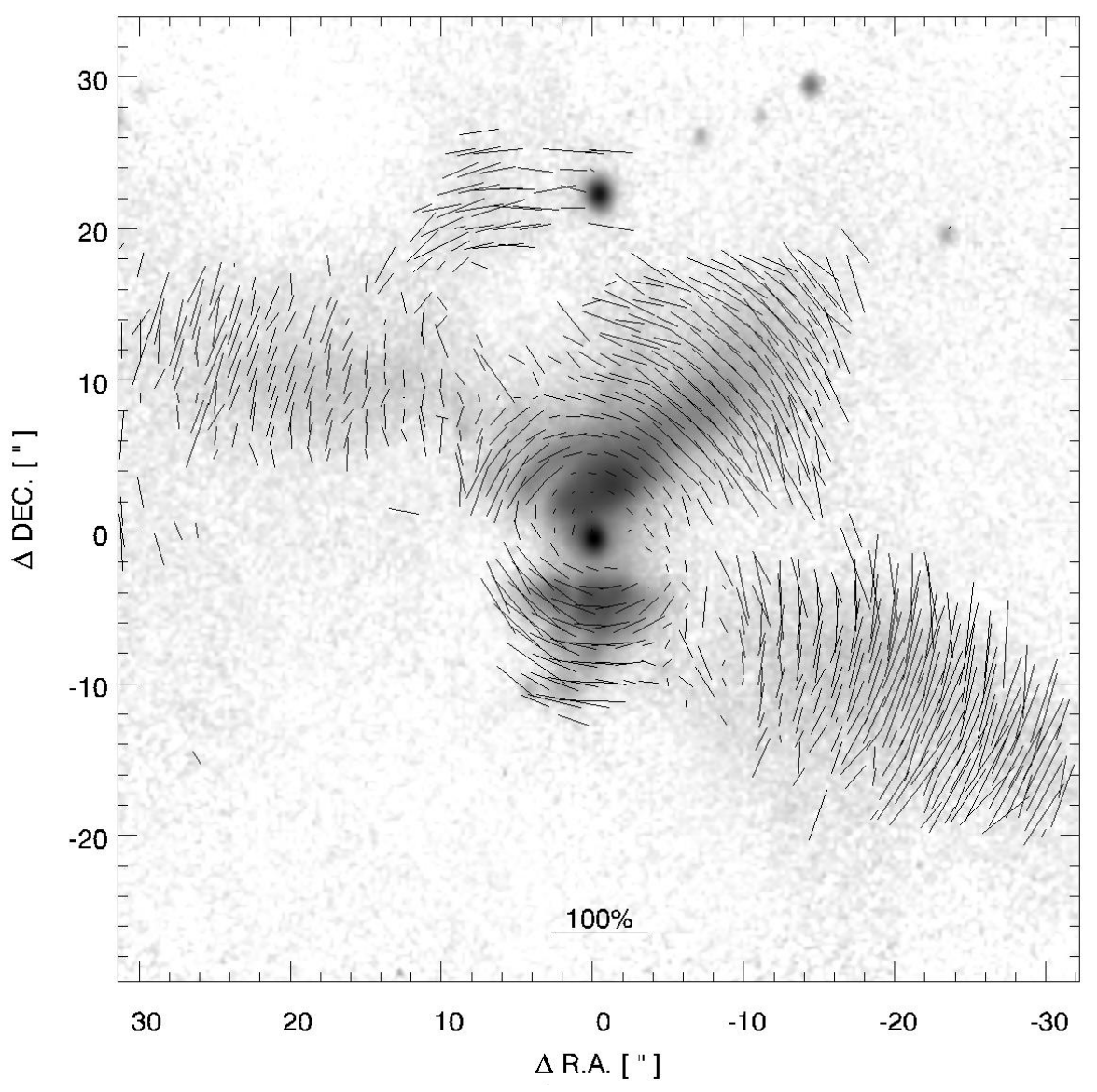

Figure 1. High-resolution K band polarization map of the optical reflection nebula Cederblad 110 IRS 4 in the Chamaeleon I dark cloud. The Cederblad 110 area is probably the youngest and most active site of low mass star formation within the Chamaeleon I cloud. ${ }^{4}$ These observations have been obtained with the near-infrared camera ISAAC at the VLT (UT1). The map is centered on the position of the illuminating source which was derived from the intersection of the perpendiculars to each polarization vector. Only polarization vectors representing polarization degrees $P_{1} \geq 10 \%$ have been considered. The slight offset of the intensity peak to the derived position of the illuminating source indicates that the source is hidden from direct view and the intensity peak is due to scattered light. The deviation from the centro-symmetry of the polarization pattern in the weak nebula located north-east of the central source might be due to additional contribution of multiply scattered radiation. See Zinnecker et al. $^{5}$ for a high-resolution JHK composite image and further details about this object. 

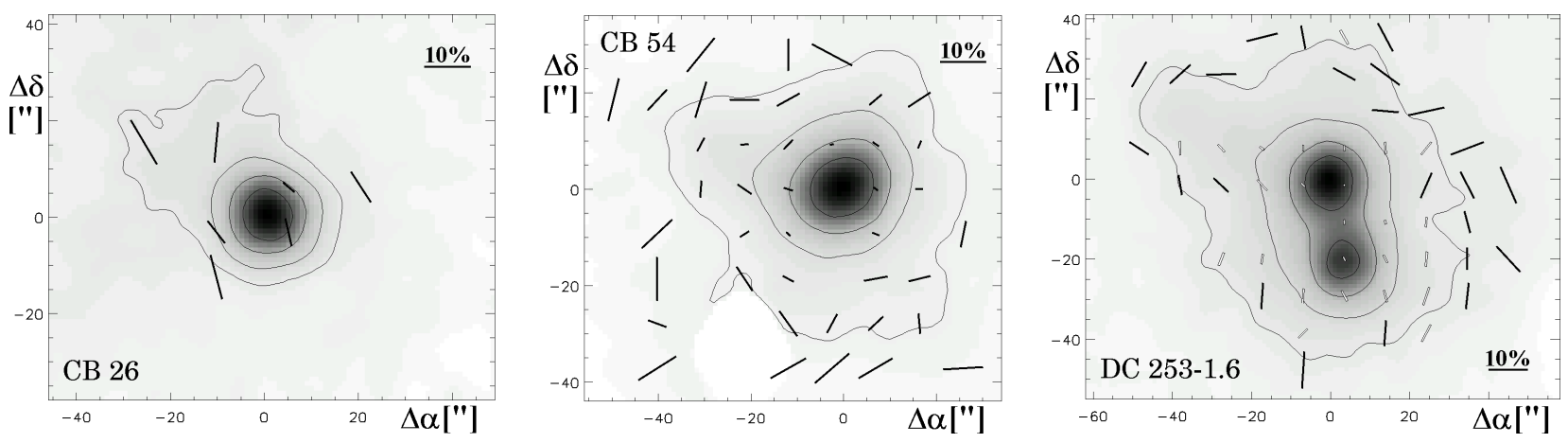

Figure 2. SCUBA $850 \mu \mathrm{m}$ maps of the Bok globules CB 26, CB 54, and DC253-1.6 with polarization vectors superimposed. The length of the vectors stands for the polarization degree and the direction gives the position angle. The data are binned over 9". Only vectors in which the $850 \mu \mathrm{m}$ flux exceeds 5 times the standard deviation and $P_{1} / \sigma\left(P_{1}\right)>3$ are plotted. The contour lines mark the levels of $10 \%, 25 \%, 50 \%$, and $75 \%$ of the maximum intensity. [taken from Henning et al. $\left.^{19}\right]$

references therein). This implies a net alignment of the grains with the magnetic field, generally with their long axes perpendicular to the field (see, e.g., Lazarian et al., ${ }^{17}$ Draine \& Weingartner ${ }^{15}$ ). Thus, the thermal emission from grains at far infrared and millimeter wavelengths is partially linearly polarized, with a polarization direction perpendicular to the magnetic field as projected onto the plane of sky. From multi-wavelength polarimetric measurements in combination with simultaneously obtained intensity maps, the magnetic field structure and strength, the dust density distribution, chemical composition, a rough estimate of the dust grain size and shape distribution, and the grain alignment rate can be derived (see, e.g., Goodman ${ }^{18}$ ). For illustration, we derive the magnetic field strength and structure in Bok globules from the submillimeter polarization patterns in Sect. 2.

\section{SUBMILLIMETER POLARIZATION MAPS OF BOK GLOBULES - MAGNETIC FIELD MEASUREMENTS}

In order to study the influence and structure of the magnetic field in the early phases of low-mass star formation, we obtained polarization maps of three Bok globules at a wavelength of $850 \mu \mathrm{m}$, using the Submillimeter Common-User Bolometer Array (SCUBA) at the James Clerk Maxwell Telescope (JCMT). Due to their relatively isolated location, Bok globules are well suited to study of the direct interplay between protostellar collapse, fragmentation, and magnetic fields since they are less affected by strong turbulence and other nearby star-forming events. The submillimeter continuum maps trace mainly the dense cores which often consist of central condensations unresolved in single-dish observations and their envelopes. We observed the following sources: CB 26 - a globule with a nearly dispersed dense core containing a source with a circumstellar disk, CB 54 - a deeply embedded young stellar cluster, and DC 253-1.6 (CG 30) - a protostellar double core.

The following similarities of the polarization patterns shown in Fig. 2 have been found:

1. The polarization degrees amount to several percent. The mean percentage polarization degrees for CB 26 , CB 54, and DC 253-1.6 are 7.3\%, 5.1\%, and 5.0\%. The corresponding $1 \sigma$ dispersions are $2.5 \%, 2.9 \%$, and $2.6 \%$.

2. In the case of CB 54 and DC 253-1.6, where we have a sufficient number of polarization vectors for further statistical analysis, we found that the polarization degree decreases towards the globule cores. The quantitative, functional dependence of this behavior is very similar. This suggests that the optical properties of the grains do not play a key role for the observed polarization decrease, but rather it is the coupling of the magnetic field to the grains that is important. 

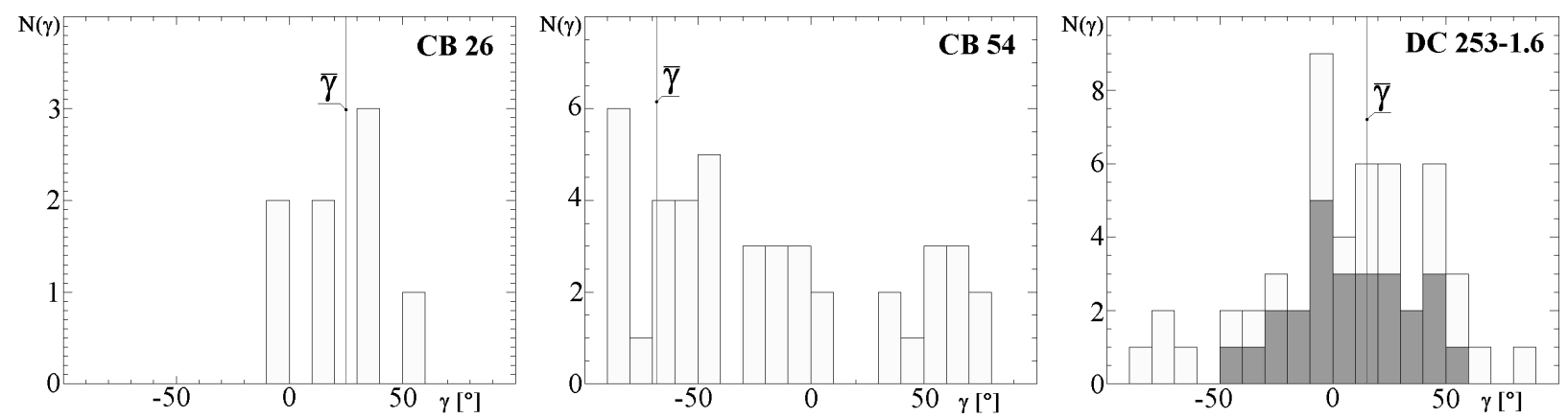

Figure 3. Histograms showing the distribution of position angles around CB 26, CB 54, and DC 253-1.6. Only data points in which the $850 \mu \mathrm{m}$ flux exceeds 5 times the standard deviation and $P_{1} / \sigma\left(P_{1}\right)>3$ have been considered. In the case of DC 253-1.6 the dark grey distribution represents the contribution of polarization vectors with $P_{1}<5 \%$ to the entire distribution. [taken from Henning et al. ${ }^{19}$ ]

Based on the work by Chandrasekhar \& Fermi (1953), the dispersion of polarization position angles is thought to be inversely proportional to the magnetic field strength. Thus, a uniform polarization pattern implies a uniform and strong magnetic field. The projected magnetic field vectors are oriented perpendicular to the direction of the polarization observed. An estimate of the magnetic field strength (in units of G) can be derived from the polarization maps as follows:

$$
B=|\vec{B}|=\sqrt{\frac{4 \pi}{3} \rho_{\text {Gas }}} \cdot \frac{v_{\text {turb }}}{\sigma_{\bar{\gamma}}} .
$$

Here, $\rho_{\text {Gas }}$ is the gas density (in units of $\mathrm{g} \mathrm{cm}^{-3}$ ), $v_{\text {turb }}$ the rms turbulence velocity (in units of $\mathrm{cm} \mathrm{s}^{-1}$ ), and $\sigma_{\bar{\gamma}}$ the standard deviation to the mean orientation angle $\bar{\gamma}$ of the polarization vectors (in units of radians; see Fig. 3).

The resulting magnetic fields, determined by the application of Eq. 1 are $B_{\mathrm{CB} 26} \approx 74 \mu \mathrm{G}, B_{\mathrm{CB}} 54 \approx 60 \mu \mathrm{G}$, and $B_{\mathrm{DC} 253} \approx 16 \mu \mathrm{G}$. The magnetic field strengths we derived are comparable to those found in molecular clouds (see, e.g., Bhatt \& Jain ${ }^{20}$ ), pre-protostellar cores, ${ }^{21}$ and other star-forming regions (see, e.g., Davis et al. ${ }^{22}$ Glenn et al., ${ }^{23}$ Itoh et al., ${ }^{24}$ Minchin \& Murray, ${ }^{25}$ Chrysostomou et al. ${ }^{26}$ Crutcher $\left.{ }^{27}\right)$. For further results of this submillimeter polarization study we refer to Henning et al.. ${ }^{19}$

\section{NEAR-INFRARED POLARIZATION OF YOUNG LOW-MASS BINARY STARS - ALIGNMENT OF CIRCUMSTELLAR DISKS}

Near-infrared (NIR) imaging and high-resolution observations provide conclusive evidence that most (if not all) low-mass stars are born in binary and multiple stellar systems. ${ }^{28-31}$ This finding suggests that binary formation is the rule and the birth of single stars the exception. The goal of the following study was to investigate whether low-mass pre-main sequence binary stars are formed by multiple fragmentation of molecular cloud cores as suggested by numerical studies ${ }^{32-36}$ or via stellar capture during close encounters. ${ }^{37}$

The fact that circumstellar disks are an inevitable means to form stars implies that the individual disks surrounding the binary components represent tracers of the binary formation mechanism. If binaries form preferentially by fragmentation, we expect their disks to be co-planar. On the other hand, the capture scenario will preferentially lead to a random distribution of disk orientations.

The scattering of emergent light from the star at the disk surface and in the lobes of the circumstellar envelope leads to a net polarization. ${ }^{39-41}$ The position angle of the polarization vector is indicative for the disk orientation. However, one has to take into account that only the projection of the disk onto the tangential plane can be measured (see Fig. 4 for illustration). Therefore, in the case of an individual binary, we cannot rule out that the disks have indeed different inclinations relative to the line of sight although the position angles of the 


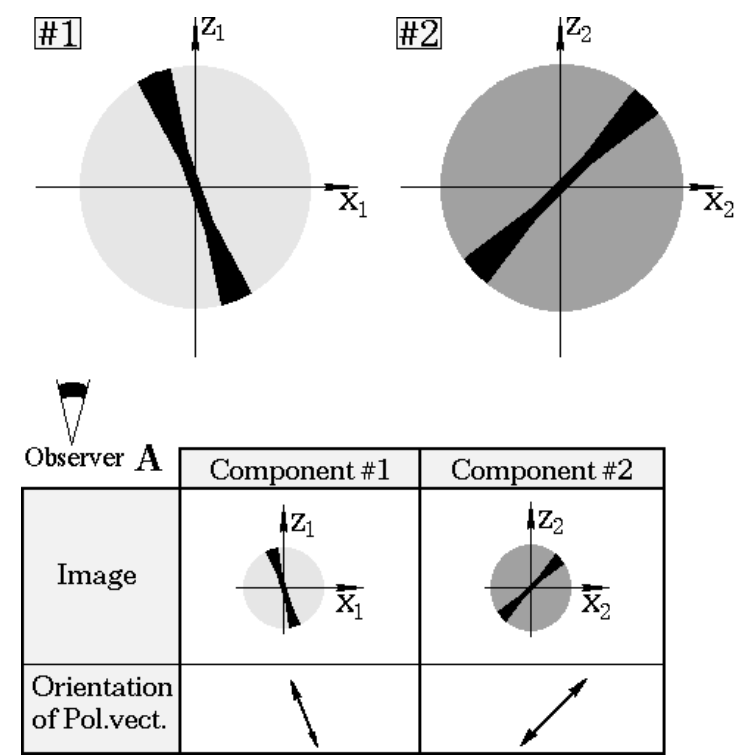

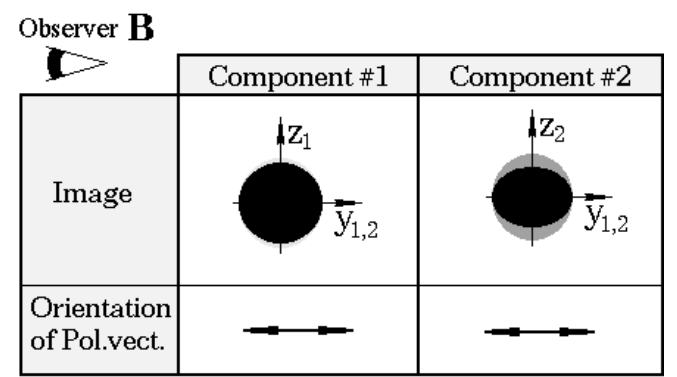

Parallel orientation of polarization vectors in spite of inclined disks !

Figure 4. Illustration of the uncertainty about the real inclination of the individual disks resulting from the performed polarization measurements. [taken from Wolf et al. ${ }^{38}$ ]

polarization of both components are similar. However, a statistical study allows us to test which hypothesis (co-planarity, random orientation) is consistent with the observed distribution of polarimetric position angles.

Using SOFI at the New Technology Telescope of ESO we measured the Ks band polarization for sample of 49 binary stars (angular separations in the range of $0.5^{\prime \prime}-5.3^{\prime \prime}$ ) which were selected from the binary surveys of Reipurth \& Zinnecker ${ }^{42}$ and Ghez et al.. ${ }^{29}$ The near-infrared excesses of the targets mostly point to the presence of disks (e.g., Kenyon et al. ${ }^{43}$ ). For a major fraction of the sample, evidence for disks is also obvious from other features (outflows, jets, Herbig-Haro objects).

The histograms of the measured linear polarizations and the position angle differences $\Delta \gamma$ based on 34 binaries are shown in Fig. 5. The remaining objects were excluded because the error of polarization ${ }^{44}$ and
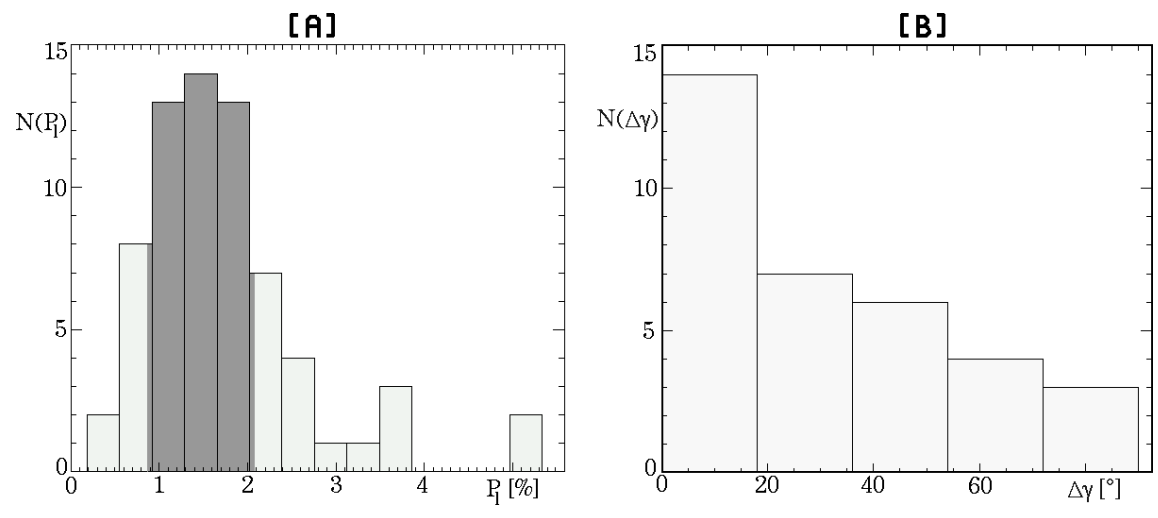

Figure 5. [A] Histogram of the polarization measured for $2 \times 34$ binary components (in case of the remaining 15 binaries at least one of the components did not show a significant polarization). The dark grey region markes the ranges in which we found a local maximum of the linear polarization in corresponding model simulations (see Wolf et al. ${ }^{38}$ for details). [B] Distribution of the position angle differences $\Delta \gamma$ of the disks in the 34 binaries. [taken from Wolf et al. ${ }^{38}$ ] 

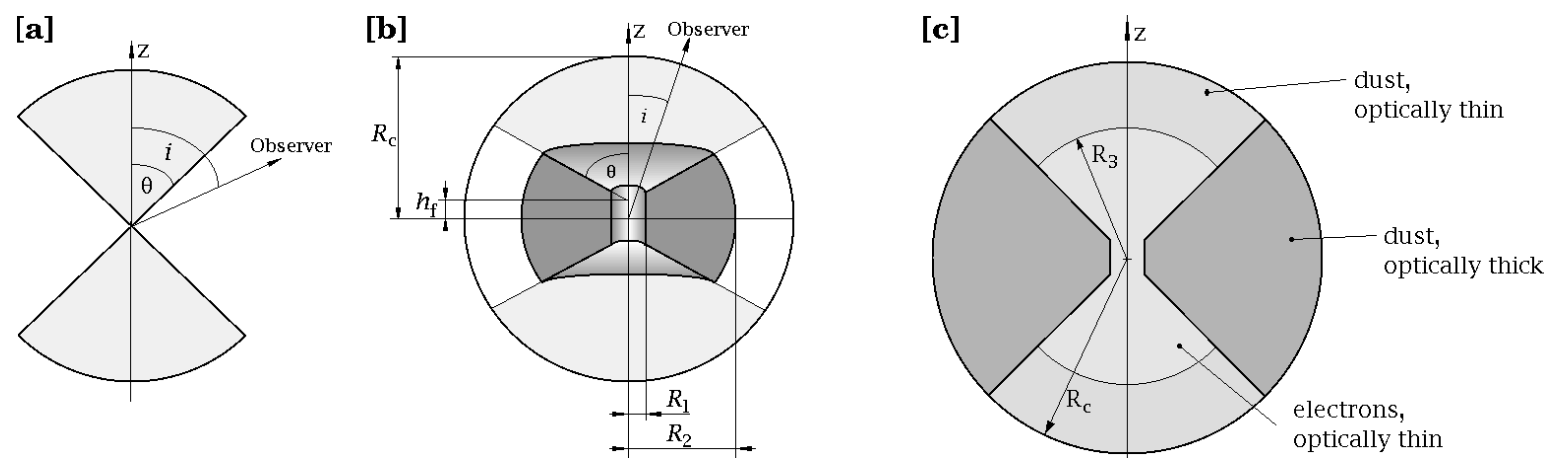

Figure 6. [a] AGN polarization model by Brown \& McLean ${ }^{50}$; [b] General model geometry considered in our AGN polarization study; [c] Model configuration for the combination of electron and dust scattering in the cones.

therefore the error of the orientation angle $\gamma$ of at least one component of the binary system was too large $\left(P_{1} / \sigma\left(P_{1}\right)<3\right)$. The distribution $N(\Delta \gamma)$ shows a strong decrease towards large angular differences (inclinations) $\Delta \gamma$. Assuming the interstellar polarization to cause this behaviour, the degree of linear polarization had to exceed 1\% (for simulation of the influence of the interstellar polarization on the results see Wolf et al. ${ }^{38}$ ). In contrast to this, the mean degree of linear polarization caused by the interstellar medium in the $\mathrm{K}$ band was found to be well below $1 \%$ in average (see, e.g., Nagata ${ }^{45}$ ). Thus, the distribution $N\left(P_{1}\right)$ reflects the intrinsic polarization of the binary components.

The measured probability distribution function $N(\Delta \gamma)$ shows a steep decrease towards large inclinations $\Delta \gamma$. We can therefore conclude that the disks in our sample are preferentially aligned, supporting the fragmentation hypothesis. Otherwhise, if stellar capture plays a role during the binary formation process, it must be followed by disk alignment processes on a timescale much smaller than the lifetime of the circumstellar disks.

\section{HIGH-RESOLUTION POLARIZATION STUDY OF AGNS}

The investigation of AGNs by polarimetric measurements provides a well-established basis for deriving their geometrical structure. The available results from observations of AGNs show that the behaviour of polarization fits in principle to the current unified model of Seyfert galaxies, as proposed by Antonucci \& Miller. ${ }^{46}$ This model is based on the assumption that all Seyfert galaxies host a Seyfert 1 type nucleus surrounded by a geometrically and optically thick torus which obscures our view of the nuclear regions for the type 2 Seyferts but not for the Seyfert 1 galaxies.

It was our aim to survey the influence of several model parameters of various AGN model geometries on the resulting polarization in the visible to near-infrared wavelength range. Using a Monte-Carlo radiative transfer code, ${ }^{47-49}$ single and multiple scattering by spherical grains and free electrons (Thomson scattering) were considered as the dominant sources of polarization.

Beginning with the most simple AGN polarization model consisting of two diametrically opposed cones ${ }^{50-52}$ (see Fig. 6[a]), we first investigated the influence of multiple scattering on the observable polarization. It was found that multiple scattering is negligible only for optical depths below $\approx 0.1$, while it has a considerable influence on the radiation characteristics at higher optical depths both in the Seyfert 1 and the Seyfert 2 case.

Second, we added a torus to the above mentioned model (see Fig. 6[b]). As the most fundamental consequence, this leads to an understanding of the phenomenological differences between Seyfert 1's and 2's. ${ }^{53-56}$ On the one hand, the torus provides the collimation of the radiation arising from a central point-like source. On the other hand, it screens the central region from the direct view (Seyfert 2 case). Having electrons in the cones and dust in the optically thick torus, ${ }^{53,57-65}$ the influence of light scattering on the torus surface was found to be marginal in contrast to the case of a torus filled with electrons. The wavelength dependence of the polarization being found for some AGNs can therefore not be explained by this model. The results for 


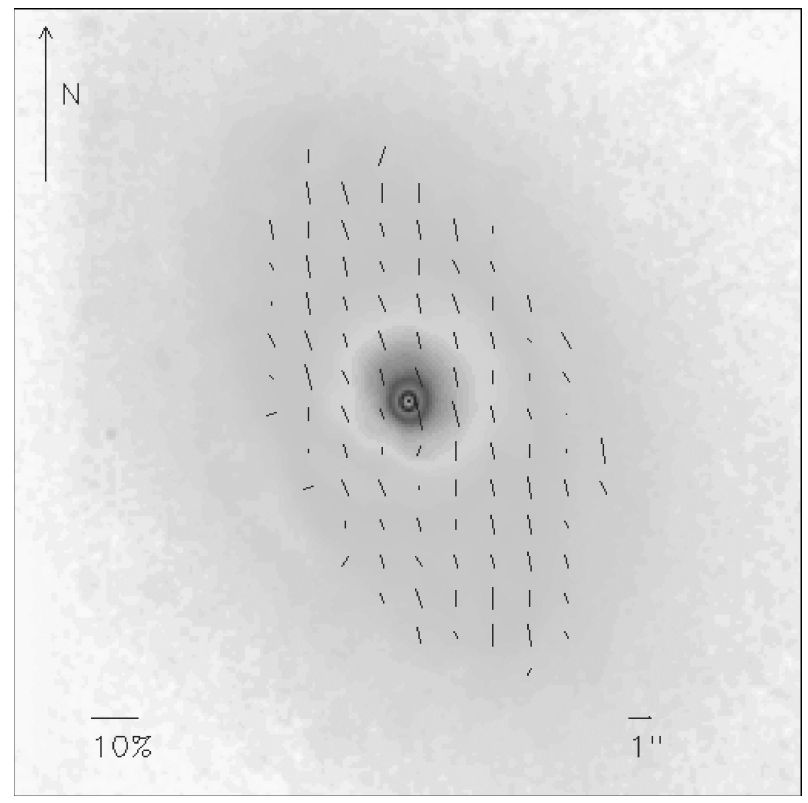

Figure 7. Intensity map with overlaied polarization pattern of the Circinus galaxy obtained with SofI/NTT at the European Southern Observatory (Filter: CO, narrow-band). Circinus is a nearby (4 Mpc), highly inclined type 2 Seyfert galaxy. [taken from Wolf et al. ${ }^{68}$ ]

the model with dust in the optically thin cones are consistent with the observations of these AGNs. ${ }^{52}$ The additional presence of electrons in the inner regions of the cones increases the absolute amount of the degree of polarization (see Fig. 6[c]). Depending on the torus size compared with the extent of the electron scattering region, the inclination of the torus may be of strong importance for the polarization degree. The inner torus radius was found to be a negligible parameter of this particular model.

If the torus has a funnel-like inner structure ${ }^{66}$ (see Fig. $6[\mathrm{~b}]: h_{\mathrm{f}}>0$ ) or a density distribution following a power law, the effective opening angle of the torus differs from the geometrical one. In both cases, this leads to a considerable change of the polarization in the Seyfert 2 case. The change of the relative intensities is noticeable for both the Seyfert 1 and 2 case.

Finally, we investigated the influence of extended central radiation sources and thermal dust re-emission from the torus surface on the polarization. The influence of an extended radiation source is not significant, while the polarization decreases with increasing dust re-emission intensity and with decreasing concentration of the reemitting region to the centre. For further results of this study we refer to Wolf \& Henning. ${ }^{67}$

\section{CONCLUSIONS}

We present submillimeter and near-infrared high-resolution polarization studies of objects representing different evolutionary stages of the star formation process: Bok globules, the optical reflection nebula Cederblad 110 IRS 4, and a sample of pre-main sequence low-mass binary stars. Based on these examples we demonstrate how polarization measurements can reveal information about these objects which cannot be derived from intensity maps alone (if at all): In the case of the Bok globules we consider the magnetic field structure and strength, while in the case of the optical reflection nebula Cederblad 110 IRS 4 we derive the location of the illuminating source. In the binary star survey the relative orientation of the circumstellar disks is considered. Finally, we discuss results from Monte-Carlo radiative transfer simulations for various model geometries of active galactic nuclei in respect of their polarimetric properties in the visible to near-infrared wavelength range. 


\section{ACKNOWLEDGMENTS}

This research was supported by the DFG grant Ste 605/10 within the program "Physics of star formation", the travel grant He1935/19-1 of the DFG, through NASA grant NAG5-11645, and through the grant DP.10633 (project 101555). R. Launhardt acknowledges financial support through NFS grant AST 9981546. JCMT is operated by the Joint Astronomy Centre on behalf of the U.K. Particle Physics and Astronomy Research Council. The presented near-infrared polarimetric data (Sect. 1, 3, and 4) have been obtained at the New Technology Telescope and the Very Large Telescope of the European Southern Observatory (ESO) and the $3.5 \mathrm{~m}$ telescope of the Calar Alto Observatory (Spain).

\section{REFERENCES}

1. B. Stecklum, M. Feldt, T. Henning, H.-U. Kaeufl, and S. Wolf, "Infrared imaging polarimetry of massive star-forming regions," in In Proc. of Galactic star formation across the stellar mass spectrum, in press, San Francisco, 2002.

2. D. Weintraub and J. Kastner, "The exciting young stellar object for the molecular outflow at the core of L1287," Astrophys. J. 411, p. 767, 1993.

3. S. Wolf, N. V. N.V., and T. Henning, "Multiple scattering of polarized radiation by non-spherical grains: First results," Astron. Astrophys. 385, p. 365, 2002.

4. P. Persi, A. Marenzi, M. Gómez, and G. Oloffson, "Sub-arcsec resolution near-infrared images of the Cederblad 110 region," Astron. Astrophys. 907, p. 916, 2001.

5. H. Zinnecker, A. Krabbe, M. McCaughrean, and et al., "A search for young solar system analogues with the VLT," Astron. Astrophys. 352, p. L73, 1999.

6. D. A. Weintraub, A. A. Goodman, and R. L. Akeson, "Polarized light from star-forming regions," in Protostars and planets IV, V. Mannings, A. P. Boss, and S. S. Russell, eds., p. 247, The University of Arizona Press, Tucson, 2000.

7. D. P. Clemens and K. E. Kraemer, "First detection of polarized dust emission from a filamentary dark cloud: Magnetic field maps," Bull. American Astron. Soc. 194, p. 47.14, 1999.

8. J. S. Greaves, A. G. Murray, and W. S. Holland, "Investigating the magnetic field structure around star formation cores," Astron. Astrophys. 284, p. L19, 1994.

9. W. A. Hiltner, "On the presence of polarization in the continuous radiation of stars. II," Astrophys. J. 109, p. $471,1949$.

10. L. Davis and J. L. Greenstein, "The polarization of starlight by aligned dust grains," Astrophys. J. 114, p. 206, 1951.

11. E. M. Purcell, "Interstellar grains as pinwheels," in The Dusty Universe, F. L. Whipple, G. B. Field, and A. G. W. Cameron, eds., p. 155, New York: Neal Watson, 1975.

12. E. M. Purcell, "Suprathermal rotation of interstellar grains," Astrophys. J. 231, p. 404, 1979.

13. T. Gold, "The alignment of galactic dust," Month. Not. Royal Astron. Soc. 112, p. 215, 1952.

14. A. Lazarian, "Physics and chemistry of the Purcell alignment," Month. Not. Royal Astron. Soc. 274, p. 679, 1995.

15. B. T. Draine and J. C. Weingartner, "Radiative torques on interstellar grains. I. Superthermal spin-up," Astrophys. J. 470, p. 551, 1996.

16. B. T. Draine and J. C. Weingartner, "Radiative torques on interstellar grains. II. Grain alignment," $A s$ trophys. J. 480, p. 633, 1997.

17. A. Lazarian, A. A. Goodman, and P. C. Myers, "On the efficiency of grain alignment in dark clouds," Astrophys. J. 490, p. 273, 1997.

18. A. A. Goodman, "The interpretation of polarization position angle measurements," in Polarimetry of the interstellar Medium, W. G. Roberge and D. C. B. Whittet, eds., ASP 97:325, San Francisco, 1996.

19. T. Henning, S. Wolf, R. Launhardt, and R. Waters, "Measurements of the magnetic field geometry and strength in Bok globules," Astrophys. J. 561, p. 871, 2001.

20. H. C. Bhatt and S. K. Jain, "Polarization measurements of stars in the region of the nearby molecular cloud MBM 12," Month. Not. Royal Astron. Soc. 257, p. 57, 1992. 
21. S. M. Levin, W. D. Langer, T. Velusamy, and T. Kuiper, "Measuring the magnetic field strength in L1498 with Zeeman-splitting observations of CCS," Astrophys. J. 555, p. 850, 2001.

22. C. J. Davis, A. Chrysostomou, H. E. Matthews, T. Jennes, and T. P. Ray, "Submillimeter polarimetry of the protostellar outflow sources in Serpens with the Submillimeter Common-User Bolometer Array," Astrophys. J. 530, p. L115, 2000.

23. J. Glenn, C. Walker, and E. T. Young, "Magnetic fields in star formation regions: 1.3 millimeter continuum polarimetry," Astrophys. J. 511, p. 821, 1999.

24. Y. Itoh, A. Chrysostomou, M. Burton, J. H. Hough, and M. Tamura, "The magnetic field structure of the DR21 region," Month. Not. Royal Astron. Soc. 304, p. 406, 1999.

25. N. R. Minchin and A. G. Murray, "Submillimetre polarimetric mapping of DR 21 and NGC 7538-IRS 11: Tracing the circumstellar magnetic field," Astron. Astrophys. 286, p. 579, 1994.

26. A. Chrysostomou, J. M. Hough, M. G. Burton, and M. Tamura, "Twisting magnetic fields in the core region of Orion molecular cloud-1," Month. Not. Royal Astron. Soc. 268, p. 325, 1994.

27. R. M. Crutcher, "Magnetic fields in molecular clouds: Observations confront theory," Astrophys. J. 520, p. 706, 1999.

28. M. Simon, A. M. Ghez, C. Leinert, and et al., "A lunar occultation and direct imaging survey of multiplicity in the Ophiuchus and Taurus star-forming regions," Astrophys. J. 443, p. 625, 1995.

29. A. M. Ghez, D. W. McCarthy, J. L. Patience, and T. L. Beck, "The multiplicity of pre-main-sequence stars in southern star-forming regions," Astrophys. J. 481, p. 378, 1997.

30. C. Leinert, A. Richichi, and M. Haas, "Binaries among Herbig Ae/Be stars," Astron. Astrophys. 318, p. $471,1997$.

31. R. Köhler and C. Leinert, "Multiplicity of T Tauri stars in Taurus after ROSAT," Astron. Astrophys. 331, p. $977,1998$.

32. I. A. Bonnell and M. R. Bate, "The formation of close binary systems," Month. Not. Royal Astron. Soc. 271, p. 999, 1994.

33. A. Burkert, M. R. Bate, and P. Bodenheimer, "Protostellar fragmentation in a power-law density distribution," Month. Not. Royal Astron. Soc. 289, p. 497, 1997.

34. A. Boss, "Collapse and fragmentation of molecular cloud cores. V. Loss of magnetic field support," Astrophys. J. 483, p. 309, 1997.

35. R. S. Klessen, A. Burkert, and M. R. Bate, "Fragmentation of molecular clouds: The initial phase of a stellar cluster," Astrophys. J. 501, p. L205, 1998.

36. M. R. Bate, "Predicting the properties of binary stellar systems: The evolution of accreting protobinary systems," Month. Not. Royal Astron. Soc. 314, p. 33, 2000.

37. J. A. Turner, S. J. Chapman, A. S. Bhattal, and et al., "Binary star formation: Gravitational fragmentation followed by capture," Month. Not. Royal Astron. Soc. 277, p. 705, 1995.

38. S. Wolf, B. Stecklum, and T. Henning, "Pre-main sequence binaries with aligned disks ?," in The Formation of Binary Stars, B. Reipurth and H. Zinnecker, eds., IAU Symp. 200, p. 295, San Francisco, 2001.

39. P. Bastien and F. Ménard, "Parameters of disks around young stellar objects from polarization observations," Astrophys. J. 364, p. 232, 1990.

40. B. A. Whitney and L. Hartmann, "Model scattering envelopes of young stellar objects. I - Method and application to circumstellar disks," Astrophys. J. 395, p. 529, 1992.

41. O. Fischer, T. Henning, and H. W. Yorke, "Simulation of polarization maps. II. The circumstellar environment of pre-main sequence objects," Astron. Astrophys. 308, p. 863, 1996.

42. B. Reipurth and H. Zinnecker, "Visual binaries among pre-main sequence stars," Astron. Astrophys. 278, p. 81, 1993.

43. S. J. Kenyon, I. Yi, and L. Hartmann, "A magnetic accretion disk model for the infrared excesses of T Tauri stars," Astrophys. J. 462, p. 439, 1996.

44. J. F. C. Wardle and P. P. Kronberg, "The linear polarization of quasi-stellar radio sources at 3.71 and 11.1 centimeters," Astrophys. J. 194, p. 249, 1974. 
45. T. Nagata, "Observation of interstellar polarization at 2.2 and 3.8 microns," Astrophys. J. 348, p. L13, 1990.

46. R. R. J. Antonucci and J. S. Miller, "Spectropolarimetry and the nature of NGC 1068," Astrophys. J. 297, p. $621,1985$.

47. S. Wolf, "MC3D - 3D Continuum Radiative Transfer, Version 2," Comp. Phys. Comm. in press, 2002.

48. S. Wolf and T. Henning, "Accelerated self-consistent radiative transfer based on the Monte-Carlo method," Comp. Phys. Comm. 132, p. 166, 2000.

49. S. Wolf, T. Henning, and B. Stecklum, "Multidimensional self-consistent radiative transfer simulations based on the Monte-Carlo method," Astron. Astrophys. 349, p. 839, 1999.

50. J. C. Brown and I. S. McLean, "Polarisation by Thomson scattering in optically thin stellar envelopes. I. Source star at centre of axisymmetric envelope," Astron. Astrophys. 57, p. 141, 1977.

51. R. W. Goodrich and J. S. Miller, "Midtail plasma flows and the relationship to near-earth substorm activity: A case study," Astrophys. J. 434, p. 82, 1994.

52. J. S. Miller, R. W. Goodrich, and W. G. Mathews, "Multidirectional views of the active nucleus of NGC 1068," Astrophys. J. 378, p. 47, 1991.

53. A. Lawrence, "Classification of active galaxies and the prospect of a unified phenomenology," Publ. Astron. Soc. Pacific 99, p. 309, 1987.

54. R. Antonucci, "Unified models for active galactic nuclei and quasars," Annual Rev. Astron. Astrophys. 31, p. $473,1993$.

55. S. Young, J. H. Hough, D. J. Axon, J. A. Bailey, and M. J. Ward, "Near-IR spectropolarimetry and modelling of NGC 1068," Month. Not. Royal Astron. Soc. 272, p. 513, 1995.

56. A. Manzini and S. di Serego Alighieri, "Dust scattering in radio galaxies," Astron. Astrophys. 311, p. 79, 1996.

57. R. Chini, E. Krügel, and E. Kreysa, "Dust emission spectra from star-forming regions," Astron. Astrophys. 167, p. 315, 1986.

58. D. B. Sanders, E. S. Phinney, G. Neugebauer, B. T. Soifer, and K. Mathews, "Continuum energy distribution of quasars - Shapes and origins," Astrophys. J. 347, p. 29, 1989.

59. R. A. Edelson and M. A. Malkan, "Far-infrared variability in active galactic nuclei," Astrophys. J. 323, p. 516, 1987.

60. E. I. Robson, W. K. Gear, L. M. J. Brown, and T. J.-L. Courvoisier, "A new infrared spectral component of the quasar 3C273," Nature 323, p. 134, 1986.

61. E. A. Pier and J. H. Krolik, "Infrared spectra of obscuring dust tori around active galactic nuclei. I. Calculational method and basic trends," Astrophys. J. 401, p. 99, 1992.

62. E. A. Pier and J. H. Krolik, "Infrared spectra of obscuring dust tori around active galactic nuclei. II. Comparison with observations," Astrophys. J. 418, p. 673, 1993.

63. A. Efstathiou and M. Rowan-Robinson, "Dusty discs in active galactic nuclei," Month. Not. Royal Astron. Soc. 273, p. 649, 1995.

64. A. Efstathiou, J. H. Hough, and S. Young, "A model for the infrared continuum spectrum of NGC 1068," Month. Not. Royal Astron. Soc. 277, p. 1134, 1995.

65. V. Manske, T. Henning, and A. B. Menshchikov, "Flared dust disks and the IR emission of AGN," Astron. Astrophys. 331, p. 52, 1998.

66. R. D. Blandford, Active galactic nuclei, Manchester University Press, Manchester, 1985.

67. S. Wolf and T. Henning, "AGN polarization models," Astron. Astrophys. 341, p. 675, 1999.

68. S. Wolf, L. Vanzi, and N. Ageorges, "How to reduce SOFI/NTT polarimetric data," tech. rep., ESO, 2002. 